Revue des études hébraïques et juives

18 | 2013

Le Proche-Orient ancien à la lumière des sciences sociales

\title{
Claude Lévi-Strauss : le judaïsme, le texte, l'ethnographie
}

Claude Lévi-Strauss: The Judaism, the Text and the Ethnography

$$
\text { קלוד לוי שטראוס: היהדות, הטקסט, האתנוגרפיה }
$$

\section{Madalina Vârtejanu-Joubert}

\section{(2penEdition}

\section{Journals}

Édition électronique

URL : https://journals.openedition.org/yod/1773

DOI : 10.4000/yod. 1773

ISSN : 2261-0200

Éditeur

INALCO

Édition imprimée

Date de publication : 1 septembre 2013

ISBN : 978-2-85831-207-8

ISSN : $0338-9316$

Référence électronique

Madalina Vârtejanu-Joubert, « Claude Lévi-Strauss : le judaïsme, le texte, l'ethnographie », Yod [En ligne], 18 | 2013, mis en ligne le 08 juillet 2013, consulté le 08 juillet 2021. URL : http:// journals.openedition.org/yod/1773; DOI : https://doi.org/10.4000/yod.1773

Ce document a été généré automatiquement le 8 juillet 2021.

\section{(i) (8)}

Yod est mis à disposition selon les termes de la Licence Creative Commons Attribution - Pas d'Utilisation Commerciale 4.0 International. 


\title{
Claude Lévi-Strauss : le judaïsme, le texte, l'ethnographie
}

\author{
Claude Lévi-Strauss: The Judaism, the Text and the Ethnography \\ קלוד לוי שטראוס: היהדות, הטקסט, האתנוגרפיה
}

Madalina Vârtejanu-Joubert

L'anthropologie du judaïsme ancien est, aujourd'hui encore, en quête de légitimité. En cela il fait figure à part dans l'étude de l'Antiquité, car l'anthropologie historique de la Grèce ou de la Rome antique sont des approches bien établies et reconnues parmi les historiens, voire au-delà. Les raisons de cette absence demeurent en grande partie énigmatiques, car, d'une part, les pères fondateurs de l'anthropologie moderne sont juifs et, d'autre part, l'anthropologie culturelle de l'Antiquité émerge simultanément avec l'anthropologie moderne après la Deuxième Guerre mondiale. Toutes les prémisses « logiques » étaient réunies pour que le judaïsme antique fût lui aussi abordé au prisme de cette nouvelle discipline. L'historiographie du judaïsme évolua pourtant différemment: non seulement la tradition de critique littéraire submergea toutes les autres approches, mais l'anthropologie historique naissante elle-même et l'anthropologie tout court ont exclu le judaïsme ancien de leurs préoccupations. Nous nous trouvons donc ici devant une triple question: comment expliquer le caractère dominant des disciplines du langage et de la littérature? Comment expliquer l'exclusion du judaïsme de l'étude anthropologique? Et enfin, comment expliquer l'exclusion du judaïsme antique du domaine de l'anthropologie historique? Des réponses partielles ont été formulées pour chacune de ces questions, des hypothèses de travail qui devraient être validées ou invalidées par une étude systématique des archives d'auteurs et par la mise en contexte historique de leurs travaux ${ }^{1}$. Parmi ces réponses, une retient en particulier notre attention, à savoir celle qui évoque la spécificité du judaisme comme objet d'étude ${ }^{2}$. Démarche scientifique légitime que celle de vouloir identifier les limites et le potentiel de chaque objet ou aire culturelle étudiés. Mais concernant l'objet qui nous intéresse ici, énoncer sa spécificité équivaut à décréter son incompatibilité avec l'étude dans le cadre de telle ou telle discipline, dont l'anthropologie ${ }^{3}$. C'est dans cette lignée que s'inscrit la position de Claude Lévi-Strauss, 
non seulement père fondateur de l'anthropologie culturelle, mais aussi savant ayant inspiré, malgré lui sans doute, un certain nombre de recherches en anthropologie du judaïsme ancien. Paradoxalement, Lévi-Strauss justifie théoriquement l'écart des sources hébraïques anciennes de son analyse structuraliste et en même temps, inspire un certain nombre de travaux qui mettent à profit la méthode structuraliste dans l'étude des textes du judaïsme ancien. Le présent article se propose de présenter ce dossier, celui du rapport de Lévi-Strauss aux sources juives/hébraïques anciennes et de relever parfois la perplexité du lecteur devant la contradiction entre les tenants théoriques et les aboutissants pratiques de la méthode lévi-straussienne.

La spécificité des textes juifs est abordée par Lévi-Strauss dans quatre textes, dont le plus récent entièrement consacré à ce sujet. Il s'agit, par ordre chronologique, du débat organisé par Paul Ricœur autour de la méthode structuraliste, publié dans Esprit en $1963^{4}$, de l'article intitulé « De la fidélité au texte », paru dans L'Homme en $1987^{5}$, et de l'article "Exode sur Exode ", paru dans la même revue l'année suivante ${ }^{6}$. Le regard que porte Lévi-Strauss est celui d'un spécialiste des mythes; c'est pour cette raison que s'avère intéressante la mise en perspective de ses propos sur le judaïsme avec ses autres analyses, notamment dans Le Cru et le cuit ${ }^{7}$, premier volume de sa monumentale série Mythologiques. La lecture de l'ensemble de ces passages ne dénote pas une évolution de la pensée lévi-straussienne à ce sujet, mais plutôt la récurrence d'un même paradoxe, celui entre le principe de la prudence comparatiste et la relativité de ce principe lorsqu'il doit être appliqué.

Commençons par l'entretien de Lévi-Strauss avec le "groupe philosophique » de la revue Esprit, entretien qui porte au fond sur le domaine d'applicabilité de la méthode structurale: est-elle universelle? Est-elle apte à rendre compte de toute pensée mythique ou, au contraire, sa pertinence se limite-t-elle au domaine américain (que Ricœur désigne comme domaine du totémisme) ? Malgré leurs divergences, Ricœur et Lévi-Strauss sont d'accord au moins sur un point : la méthode structurale ne peut pas, en l'état, aider à comprendre certains pans de la pensée humaine. Leurs arguments se rejoignent partiellement.

Ricœur campe sa critique depuis les positions de sa propre méthode, l'herméneutique, considérant qu'une incompatibilité intrinsèque entre des objets différents limite la pertinence de la méthode structurale :

européen, sémitique, aient permis $\mathrm{t}$

«Pensez-vous que ce soit un accident que justement les fonds préhellénique, indo-

Strauss sont plus complexes. Tout en acquiesçant à la remarque de son préopinant, il

Les arguments de Lévi-

précise : «L

e principe de la différence qu'il postule ne m'apparaît pas être dans les pensées

elles-mêmes, mais dans les situations variées où l'observateur se trouve vis-à-vis de ces

pensées. » Il s'agit ici d'une thématique chère à Lévi-Strauss, celle du point de vue de

l'observateur: à ses yeux, il est impossible pour le chercheur de se pencher sur sa propre société, la posture de l'objectivité étant compatible uniquement avec le «dehors » et absolument pas avec le « dedans »:

Mais parce que là, nous sommes prisonniers de la subjectivité, nous ne pouvons pas,

à la fois, essayer de comprendre les choses du dehors et du dedans; et nous ne pouvons les comprendre du dedans que si nous sommes nés dedans, que si nous sommes effectivement dedans. L'entreprise qui consiste à essayer de transporter si je puis dire - une intériorité particulière dans une intériorité générale me semble d'avance compromise ${ }^{8}$. 
Il déclare s'abstenir « soigneusement » d'appliquer sa méthode aux textes mythiques de sa propre tradition. En réalité, Lévi-Strauss tentera cette aventure, de manière sporadique dans ses Mythologiques et de manière systématique dans son article sur la circoncision de 1988. Ce texte, sur lequel nous reviendrons, donne l'impression d'un auteur apprenti sorcier, inquiet des conséquences de sa méthode et tentant d'en diminuer au maximum la portée épistémologique.

Une première distinction qu'établit Lévi-Strauss est donc celle entre la pensée qui fait l'objet de l'observation et l'observateur. Il établit une autre distinction, plus discutable elle, entre la pensée en soi et les supports de transmission de cette même pensée. C'est une des raisons qu'il invoque à l'appui de son refus d'inclure l'Ancien Testament dans ses analyses et un argument pour qualifier de «jeu» l'ouvrage de l'anthropologue anglais Edmund Leach, Lévi-Strauss in the Garden of Eden. De la même manière qu'il met en motto de son «Exode sur l'Exode » une vieille acception de ce terme, puisée dans le
Dictionnaire
de
Trévoux
(1704):

Parmi les Latins, exode a été pris dans un autre sens. C'était parmi eux à peu près ce que la Farce est parmi nous. » Pourquoi toute approche structurale de l'Ancien

$\ll$

Testament

elle un amusement, une plaisanterie, un jeu ? Voici le premier volet de sa réponse, avec seraitnos soulignements :

D'abord, parce que l'Ancien Testament, qui met certainement en œuvre des matériaux mythiques, les reprend en vue d'une autre fin que celle qui fut originellement la leur. Des rédacteurs les ont, sans nul doute, déformés en les interprétant ; ces mythes ont donc été soumis, comme dit très bien M. Ricœur, à une opération intellectuelle. Il faudrait commencer par un travail préliminaire, visant à retrouver le résidu mythologique et archaïque sous-jacent à la littérature biblique, ce qui ne peut évidemment être l'œuvre que d'un spécialiste".

Ce court passage pose des questions épistémologiques extrêmement sérieuses. Qu'est-ce qu'un «mythe» et qu'est-ce qu'un «résidu mythique»? Comment reconnait-on un matériau mythique « déformé »?

La problématique de la multiple rédaction de l'Ancien Testament/Bible hébraïque n'est pas nouvelle à l'époque de Lévi-Strauss et on peut supposer qu'il était au fait au moins de ce principe sinon des acquis de l'exégèse biblique. Mais ce n'est pas cet énoncé général qui peut nous aider à déchiffrer ce passage qui exclut la méthode structurale du domaine biblique et juif. Malheureusement, jamais Lévi-Strauss ne donnera plus de détails sur la manière dont il conçoit l'interdépendance subsistant entre un palimpseste textuel - en occurrence la Bible - et l'analyse structurale. Nous en sommes réduits aux hypothèses ${ }^{10}$.

À défaut d'une définition théorique du « résidu mythique $»^{11}$, on peut penser que cette phrase fait allusion à une opinion scientifique déjà établie, celle de l'historicisation des mythes hébreux. Selon cette interprétation, les mythes qui, à l'origine, prenaient comme support les phénomènes de la nature sont associés à un événement de la vie d'un peuple. Par exemple, à la fête de l'équinoxe de printemps célébrant le renouvellement de la nature, on a associé la sortie d'Égypte et de cette synthèse est née la Pâque/Pessah. De même a-t-on associé, à l'époque hellénistique, la fête de la lumière au moment du solstice d'hiver à la reconquête de l'indépendance nationale sous les Maccabées symbolisée par le miracle de la lumière lors de la purification du Temple, Hanouka. Il est cependant plus difficile de distinguer entre une phase naturelle et une phase historicisante des mythes dans le cas, par exemple, du récit de la cosmogonie 
biblique ou dans celui de la sortie d'Abraham d'Our en Chaldée. Devrait-on par conséquent distinguer au préalable entre un mythe et un récit? Lévi-Strauss semble attaché à cette distinction :

Même dans la mythologie dont traitera presque entièrement mon prochain livre, je veux dire celle de l'Amérique tropicale, j'aperçois des niveaux hétérogènes. Aussi, je préfère laisser de côté certains textes, au moins provisoirement, parce que leur organisation interne semble relever d'autres principes; il existe en Amérique du Sud une littérature presque romanesque mêlée aux mythes qui, peut-être, est passible de l'analyse structurale, mais alors d'une analyse structurale transformée et affinée, que je n'ose aborder pour le moment ${ }^{12}$.

L'analyse structurale suppose donc que le mythe soit préservé dans sa forme littéraire pure. Un roman, une saga, une légende ne sont pas justiciables, selon Lévi-Strauss, de la même méthode que le mythe, car l'identification des oppositions binaires serait impossible. Pour qu'un sens se dégage du mythe, il faut qu'on puisse identifier la position de chaque élément par rapport à son opposé, par rapport à ce qu'il n'est pas. Or, Lévi-Strauss attribue cette propriété uniquement au mythe, qu'il entend sous l'angle de la forme d'expression - du genre littéraire en somme. Pour cette raison, il n'inclut pas dans ses analyses de la transformation des mythes sur le continent américain toute une littérature de l'Amérique centrale qui lui semble relever non seulement d'un genre différent, mais aussi d'une organisation étatique absente dans les sociétés tribales à dominante "mythologique». Son disciple Pierre Clastres va développer cette idée en théorisant sur le rapport existant entre les formes d'expression littéraire et la naissance de l'État ${ }^{13}$.

La question de la pureté littéraire des mythes comme condition préliminaire à toute analyse structurale débouche sur la question des rapports entre approche synchronique et approche diachronique. Lévi-Strauss conçoit un certain ordre épistémologique au sein duquel l'étude typologique précède nécessairement l'étude historique :

Je dis souvent à mes étudiants qu'il n'y aurait pas eu un Darwin s'il n'y avait pas eu d'abord un Linné ; on n'aurait pas pu poser le problème de l'évolution des espèces si on n'avait pas commencé par définir ce qu'on entend par espèce et par faire une typologie ${ }^{14}$.

On comprend pourquoi la notion d'hybridité littéraire et de palimpseste pose à Lévi-Strauss un problème épistémologique de premier ordre. La question qui se pose aux biblistes en retour est de savoir si postuler une forme finale du texte et lui appliquer une analyse structurale est pertinent. De quoi une telle analyse est-elle le reflet? Si Lévi-Strauss analysait les mythes pour nous dévoiler une forme de pensée particulière, distincte du raisonnement causal, quelle est la portée d'une analyse structurale de tout récit pris dans sa forme finale? Des biblistes se sont exercés à l'analyse structurale des récits bibliques ${ }^{15}$, mais ces questions attendent encore leurs hypothèses explicatives.

Les conflits entre mythe et récit d'une part, entre structure et histoire d'autre part, forment le premier volet des arguments de Lévi-Strauss à l'encontre d'une étude structurale de la Bible. Il existe un deuxième volet argumentatif, formulé lui aussi dans l'entretien autour du groupe philosophique de la revue Esprit ${ }^{16}$ :

[...] Il me semble qu'une entreprise de cet ordre implique une sorte de cercle vicieux qui tient au fait qu'à mes yeux - et c'est peut-être là un point de désaccord avec M. Ricœur -, les symboles - pour reprendre un terme qu'il affectionne - n'offrent jamais une signification intrinsèque. Leur sens ne peut être que « de position » et, 
par conséquent, il ne nous est pas accessible dans les mythes mêmes, mais par référence au contexte ethnographique (n. s.), c'est-à-dire ce que nous pouvons connaître du genre de vie, des techniques, des rites et de l'organisation sociale des sociétés dont nous voulons analyser les mythes. Dans le cas de l'ancien judaïsme (n. s.), nous nous heurterions à une situation paradoxale, puisque le contexte ethnographique fait presque entièrement défaut (n. s.), sinon celui, précisément, qu'on peut extraire des textes bibliques. Toutes nos hypothèses reposeraient donc sur une pétition de principe. Ce que je viens de dire pour la Bible peut être étendu à d'autres sources mythologiques: les grands textes de l'Inde ancienne, les classiques de la protohistoire japonaise, Kojiki et Nihongi, et bien d'autres choses. Il y a donc une masse considérable de matériaux que je me suis abstenu d'attaquer, je le répète: d'une part en raison de l'absence de contexte ethnographique et d'autre part, parce qu'ils réclameraient une exégèse préalable que l'ethnologue n'est pas qualifié pour entreprendre.

Lévi-Strauss adopte ici une position minimaliste en ce qui concerne les textes du judaïsme ancien, sous-entendu la Bible hébraïque. Il convoque ainsi un débat de fond, mais en bonne mesure aporétique: aucune règle formelle n'a pu être établie pour normaliser l'interprétation historique des textes antiques. Cela ne revient pas nécessairement à dire que ceux-ci n'ont aucun ancrage historique. Lévi-Strauss semble regretter l'impossibilité de les confronter aux observations sur le vif d'un enquêteur ethnographe. Dans un autre article, « De la fidélité au texte » (1987), l'anthropologue oppose non seulement le domaine antique de l'historien au domaine contemporain de l'ethnologue, mais aussi le domaine juif au domaine grec et romain :

Michael P. Carroll $\left(1985,775^{17}\right)$ se trompe quand il m'attribue l'idée que seuls les mythes provenant des sociétés naguère appelées par moi «froides » peuvent faire l'objet d'une analyse structurale. J'ai dit tout autre chose: l'analyse structurale requiert un contexte ethnographique indépendant de la matière mythique elle-même. J'écarte pour cette raison l'Ancien Testament - pratiquement notre seule source sur les anciens Hébreux - où tout est mélangé, de sorte qu'on peut toujours douter si les informations d'allure ethnographique ne font pas partie du matériel mythique qu'elles devraient servir à éclairer. Mais, pour se limiter à deux exemples, la Grèce et la Rome anciennes n'étaient certainement pas des sociétés " froides ». Nous possédons toutefois sur elles des informations ethnographiques très riches et indépendantes de leurs mythes dont G. Dumézil pour Rome, J.-P. Vernant, M. Détienne, P. Vidal-Naquet et d'autres pour la Grèce, conduisent dans le plus pur esprit structural des analyses exemplaires ${ }^{18}$.

Cette phrase est tout aussi déconcertante que la citation précédente. Car peut-on parler d'information " ethnographique » en ce qui concerne la Grèce et Rome ? Et de surcroît, d'une information totalement "distincte » des "mythes"? C'est là un propos heuristique difficilement défendable ${ }^{19}$. Par ailleurs, le projet de ces anthropologues du monde antique semble différent de celui de Lévi-Strauss qui voit dans l'apport de l'ethnographie une confirmation ou non des données empiriques contenues dans les mythes. Prenons par exemple Vidal-Naquet et son «Avant-propos» au Chasseur noir. Tout en reconnaissant sa dette envers le structuralisme, son projet n'est pas d'étudier les mythes en soi, mais d'établir des rapports entre les formes de pensée et les formes de société : «Quoi de plus abstrait, en principe, qu'une théorie de l'espace, quoi de plus concret qu'une bataille victorieuse ?» Ou encore : "Telle a, en effet, été mon ambition: faire communiquer ce qui ne communique pas naturellement, selon les critères habituels du jugement historique [...] $»^{20}$.

Pour revenir à la Bible, l'argument portant sur l'absence de source alternative n'est pas défendable, car déjà à l'époque - les années 1960 - l'épigraphie sémitique avait pris son 
essor et les manuscrits de la mer Morte commençaient à être connus. De surcroît, la Bible elle-même n'est pas un corpus monolithique, mais multiforme, renfermant des documents de différentes époques qui, par endroits, se recoupent ou se commentent les uns les autres. Naviguer entre surinterprétation et hypercritique est une entreprise difficile, mais dont il n'est pas sage de faire l'économie. Des tentatives de sortir de ce cercle vicieux ont été couronnées de succès et nous pensons particulièrement à l'œuvre de Mary Douglas ${ }^{21}$. Pour l'heure il nous semble intéressant de nous attarder sur la manière dont Lévi-Strauss applique par ailleurs ce principe qu'on pourrait appeler "ethnographique ». On constate que ses ouvrages sont souvent accompagnés d'un appendice illustrant et répertoriant les animaux et les plantes qui figurent dans les mythes analysés. Mais le rapport du mythe à la réalité est hautement complexe et il s'avère qu'en règle générale,

[...] le système mythologique possède une autonomie relative par rapport aux autres manifestations de la vie et de la pensée du groupe. Toutes sont jusqu'à un certain point solidaires, mais cette solidarité ne résulte pas dans des rapports rigides, imposant des ajustements automatiques entre les niveaux. Il s'agit plutôt de contraintes à long terme, dans les limites desquelles le système mythologique peut, en quelque sorte, dialoguer avec lui-même et s'approfondir dialectiquement ${ }^{22}$.

Les mythes ne reflètent pas automatiquement la réalité sociale et le premier volume des Mythologiques foisonne d'exemples en ce sens, Lévi-Strauss étant soucieux de relever cette caractéristique de la matière étudiée. Notons un seul de ces exemples : le passage du continu au discret, symbole du passage de la nature à la culture, dans les mythes Tikopia et Ojibwa. Chez les Tikopia, les ancêtres avaient réussi à garder uniquement quatre plantes totémiques lorsque les innombrables nourritures furent volées par un dieu étranger. Le nombre quatre correspond réellement à celui des clans et le mythe " doit franchir, mais à grand prix, le fossé qui sépare l'imaginaire du vécu " (p. 61). Chez les Ojibwa en revanche, "pour que les 5 grands clans dont ils croient leur société issue puissent se constituer, il fallut que 6 personnages surnaturels ne fussent plus que 5 , et que l'un d'eux fût chassé ». Seulement « les 5 clans primitifs ne sont pas plus réels que les 6 êtres surnaturels ». En conclusion, dans le cas des Tikopia « on va du mythe à la réalité » et dans le cas des Ojibwa " on ne sort pas du mythe » (p. 62). Cet exemple illustre bien l'ambiguïté des rapports entre mythe et réalité sociale, mais aussi la posture paradoxale du savoir ethnographique : il sert non seulement à vérifier les correspondances, mais surtout à souligner les divergences entre ces deux domaines de l'existence humaine. Mais l'absence de contexte ethnographique affecterait-elle fondamentalement l'analyse des mythes? Dans l'exemple invoqué ici, la confrontation avec la réalité aide à comprendre pourquoi les Tikopia partent de l'infini pour aboutir à 4 et pourquoi les Ojibwa se contentent d'un passage de 6 à 5 : le passage de l'imaginaire au vécu est plus ou moins important quantitativement. Mais en dehors de ce corollaire, l'analyse structurale n'est pas dépendante de la confrontation avec la réalité ethnographique; au contraire, un certain nombre d'invariants sont ainsi mis en lumière, comme le personnage du médiateur ayant un défaut physique, ce qui est un trait récurrent des mythes amérindiens, mais aussi des autres cultures:

Aveugles et boiteux, borgnes ou manchots, sont des figures mythologiques fréquentes par le monde et qui nous déconcertent parce que leur état nous apparaît comme une carence. Mais, de même qu'un système rendu discret par soustraction d'éléments devient logiquement plus riche, bien qu'il soit numériquement plus pauvre, de même les mythes confèrent souvent aux infirmes et aux malades une signification positive : ils incarnent des modes de la médiation ${ }^{23}$. 
Dans un autre chapitre, Lévi-Strauss explique la signification du chromatisme du poison en partant des mythes amérindiens pour aboutir à la légende de Tristan et Iseut :

Nous n'irons pas jusqu'à suggérer qu'Iseut est réductible à la "fonction de sarigue ». Mais, que l'analyse des mythes sud-américains nous ait amené à faire du poison de pêche ou de chasse une variante combinatoire du séducteur, empoisonneur de l'ordre social, et qu'entre nature et culture, l'un et l'autre sont apparus comme deux modalités du règne des petits intervalles, est bien pour convaincre que le philtre d'amour et le philtre de mort sont interchangeables pour d'autres raisons que celles tirées de la simple opportunité et donne à réfléchir sur les causes profondes du chromatisme de Tristan ${ }^{24}$.

Enfin, dans le dernier chapitre de Le Cru et le cuit il analyse non seulement des mythes, mais des coutumes populaires liées au mariage en France, en Afrique et dans d'autres civilisations, coutumes dont la signification se déploie en situant l'analyse à un niveau élevé de généralisation, au-delà du domaine empirique et ethnographique ${ }^{25}$.

Tout ceci nous amène à l'approche théorique qui fonde chez Lévi-Strauss la comparaison. Sa position est clairement formulée dans son article de 1988, «Exode sur Exode », à savoir " généraliser d'abord et comparer ensuite »:

[...] comparer non des rites qui superficiellement se ressemblent, mais qui diffèrent au point qu'à l'époque de Frazer, les ouvrages d'ethnologie les auraient traités séparément: le rite de circoncision à propos des mutilations corporelles, le rite d'imposition de l'étui à propos du costume ou bien des parures et des ornements. Derrière ces différences de surface, seule une critique interne permet d'atteindre un schème invariant. On se demande à quelles conditions plusieurs usages, mythes ou rites, convenablement redéfinis, pourraient être mutuellement convertibles. Et c'est cette invariance découverte à un niveau profond qui autorise et légitime le travail de comparaison. Chez Frazer (pour l'œuvre monumentale duquel rien ne peut ébranler notre admiration), un comparatisme à fleur de peau pousse à des généralisations souvent prématurées. Je procède à l'inverse : généraliser d'abord et comparer ensuite ${ }^{26}$.

C'est l'appartenance des deux rites à un schème invariant qui autorise leur comparaison: ajout ou retrait d'un prépuce culturel. Ce rite se concrétise par l'imposition d'un étui pénien chez les Bororo et par la circoncision chez les Hébreux et « répond au même projet : marquer la verge d'un signe culturel ».

delà de celui générique de parfaire culturellement la distinction entre le masculin et le

Quel sens donner à ce rite, au-

féminin? Lévi-

27 .

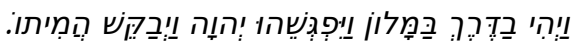

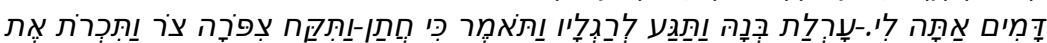

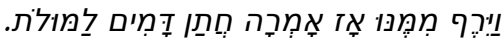

Or, en chemin, à la halte, le Seigneur l'aborda et chercha à le faire mourir. Séphora prit un silex, coupa le prépuce de son fils et lui toucha les pieds en disant : «Tu es pour moi un époux de sang. " Et il le laissa. Elle disait alors "époux de sang » à propos de la circoncision. 
Plusieurs mots et expressions font difficulté, à commencer par les pronoms personnels qui ne désignent pas explicitement leur référent, pour continuer avec l'attaque meurtrière de Dieu envers le personnage principal et finir avec la mystérieuse expression " époux de sang ». Chacune de ces difficultés trouve son pendant dans le cas du rituel bororo car les récits des informateurs à leur tour ne sont pas univoques, ni limpides. Les témoignages se comportent ici comme un texte ancien, avec ses obscurités et ses variantes.

Dans la religion bororo, lors de la remise de l'étui pénien « on conduit le parrain sur la place du village, on lui remet le bourgeon en prononçant des paroles rituelles que les informateurs traduisent : "Celui-ci, celui-ci même sera ton épouse" ${ }^{28}$

. Il est évident que le texte biblique ancien et le « texte » bororo moderne soulèvent les mêmes perplexités : qui est l'épouse dans la phrase du parrain bororo et qui est «l'époux de sang » de Séphora? Aucune unanimité historiographique n'a été acquise ni dans un cas, ni dans l'autre. On retient cependant, avec Lévi-

Strauss, que les deux sourc

29.

Enfin, les deux rituels font vraisemblablement partie intégrante d'un processus d'initiation dont une des étapes serait l'attaque du novice par un être divin : d'une part, la circoncision du fils de Séphora intervient après l'attaque meurtrière de Dieu contre lui et, d'autre part, l'imposition de l'étui pénien chez les Bororo intervient après la confrontation rituelle entre les novices et le monstre aije

. La remarque de Lévi-

Strauss à ce propos suggère le processus de rationalisation des textes mythologiques

La correspondance avec Exode IV serait complète si l'on pouvait imaginer qu'au cours de très anciens rites d'initiation, le jeune Hébreu était confronté à un monstre que les rédacteurs de la Bible auraient en quelque sorte théologisé» ${ }^{30}$.

On l'aura compris, la méthode comparatiste de Lévi-Strauss part de l'identification de schèmes invariants et rejette l'empirisme superficiel et simplificateur. Si le retrait du prépuce et l'imposition de l'étui pénien sont comparables, c'est parce que, en tant que "pratiques inverses l'une de l'autre », ils représentent des "variantes combinatoires » d'un schème unique, identifiable seulement si on va au-delà des apparences. Ces deux pratiques appartiennent au "même champ sémantique » et c'est uniquement cette appartenance qui « autorise à les comparer " ${ }^{31}$. Mais cette comparaison "à la source » est complétée dans le cas précis qu'analyse ici Lévi-Strauss par la comparaison des interrogations et des difficultés d'interprétation suscitées par les deux corpus - le texte biblique et les récits des informateurs bororo:

Quand on les superpose, elles reconstituent sur un nouveau plan cette identité sous-jacente qu'une démarche hypothético-déductive avait permis de postuler : à des exégètes qu'éloignent leurs domaines d'étude respectifs et leur formation intellectuelle, l'interprétation de rites différents pose, on l'a vu, les mêmes questions ${ }^{32}$.

Comment expliquer cette double coïncidence? Lévi-Strauss est à ce sujet, plus que prudent, déconcertant le lecteur par ses rétractations.

on tirer de ce rapprochement ? Certainement pas que les difficultés du texte ancien éclairent les aspects énigmatiques du rite contemporain ou le contraire. Le

Quelles conséquences pourra-tparallélisme que j'entends souligner n'est probablement qu'une curiosité. 
Que conclure de ce parallèle ? Rien sans doute, sinon que dans l'ordre des représentations collectives, il permet d'observer un phénomène non dépourvu d'analogie avec un autre que mutatis mutandis les amateurs de kaléidoscope se plaisent parfois à imaginer ${ }^{33}$.

Ne nous méprenons cependant pas. À la manière du rabbin maniant le spoudogeloion ${ }^{34}$, Lévi-Strauss mélange dans un même propos le sérieux et le dérisoire. Dans la dernière phrase de son article, il considère sa démarche comme la possibilité d'« offrir une sorte de confirmation expérimentale de principes théoriques et méthodologiquesqu'on n'a pas oubliés en écrivant cettefantaisie $»^{35}$

Strauss avance des explications qui devront faire réfléchir davan études juives, antiques ou modernes. Son hypothèse pour exp schème invariant supportant la comparaison fait appel à une l'autonomie de la pensée par rapport aux autres composantes fonctionnement au moyen de permutations entre des éléme

. Loin de vider complètement sa démarche de sens, Lévi-

si un enseignement s'en dégage, il se réduira à la constatation souvent faite que dans le domaine des croyances religieuses et des actions rituelles où la pensée, relativement affranchie des contraintes du milieu, parait avoir le champ libre, elle reste néanmoins soumise à des lois propres. La récurrence des mêmes problèmes d'interprétation, posés par des sociétés fort éloignées dans le temps et dans l'espace, vient donc appuyer l'hypothèse que des cadres logiques astreignent le pouvoir créateur de l'esprit à des cheminements obligés ${ }^{36}$.

Ces cadres logiques contraignants font en sorte que même si cela a peu de chances de se produire, il suffit que les petites pièces translucides emprisonnées derrière le verre soient

en nombre fini

pour qu'on puisse au moins rêver qu'après des secousses ou des rotations plus ou moins nombreuses imprimées au tube, la même configuration réapparaîtra, ou bien une configuration symétrique avec une autre, que ce soit par la forme ou par les couleurs. Nonobstant d'énormes écarts de temps et de lieux, rien n'exclut non plus que des configurations identiques apparaissent dans les mœurs, les croyances, les mythes et les rites,

si les éléments de la combinatoire

à quoi les hommes dépensent une bonne partie de leur activité mentale

n'existent pas en nombre illimité37. («Exode sur Exode », p. 21)

En ce qui concerne le comparatisme en aval, celui visant les questionnements des chercheurs, Lévi-Strauss formule là aussi des hypothèses dignes d'intérêt pour les hébraïsants. Pour expliquer les obscurités du passage biblique étudié, il adopte une des positions classiques chez les antiquisants, mettant celles-là sur le compte d'un rédacteur qui ne comprenait probablement plus une tradition dont ne subsistaient que des bribes, ou qu'il a délibérément laissée dans le vague parce qu'elle heurtait les idées

. Si, pour la Bible, l'éloignement dans le temps et le processus de rédaction sont un recours habituel pour rendre compte des difficultés textuelles, ces justifications ne peuvent pas être invoquées dans le cas des ambiguïtés des récits bororo. La multiplicité des variantes s'explique autrement : « Force est donc de reconnaître que les sages bororo auxquels on eut

recours 
ne comprenaient pas tous leurs rites de la même façon ${ }^{39}$

. Synchronisme des variantes qui s'explique à son tour par une approche dynamique du

rite :

À supposer - mais rien n'y oblige - que le rite de l'étui ait eu jadis pour les Bororo une signification non équivoque, nous avons dû constater qu'au cours des temps, cette hypothétique signification première a éclaté et qu'elle en a engendré plusieurs. Un système rituel qui se réalise hic et nunc parmi d'autres possibles conserve un dynamisme interne. Il engendre à son tour des significations également possibles qui toutes, ou certaines d'entre elles, s'actualisent dans l'esprit des interprètes : ceux du cru et ceux $\mathrm{du}$ dehors. Or, chez les uns et les autres, ces significations qu'on pourrait dire elles aussi précontraintes viennent se couler dans le même moule ${ }^{40}$.

Le rite est abordé ici comme un texte et, finalement, comme un mythe. Sujet à des

ci obéissent aux mêmes lois $c$ interprétations, celles-

associant le structuralisme au fonctionnalisme. Mary Douglas a pleinement et sans préalable abordé les sources bibliques, mettant en parallèle « corps physique » régi par les règles de pureté et « corps social » régi par les menaces externes ou internes d'anéantissement. L'œuvre de Mary Douglas et ses fondements théoriques ne font pas l'objet de cet article et nous n'entrerons pas dans les détails. Mais il fallait en faire mention pour montrer à la fois le prolongement de la méthode lévi-straussienne et son dépassement

. Sans « faire école » à proprement parler, les travaux de Mary Douglas sont devenus classiques parmi les hébraïsants et les biblistes 42 .

Pour résumer la démarche de Lévi-Strauss dans le domaine du judaïsme antique, c'est toujours le mot paradoxe

qui s'impose. Tout d'abord celui du refus programmatique de les aborder, pour les raisons développées ci-

dessus, et le fait de les abo théorique de s

\section{NOTES}

1. Lévi-Strauss formule dans une interview au Nouvel Observateur, 5 juillet 1980, deux hypothèses explicatives à la prééminence de chercheurs juifs dans le domaine de la sociologie et de l'anthropologie :

NO : Dans ses Réflexions, Sartre prétendait en substance que le juif est spontanément ethnologue. Et, de fait, vos pères en cette discipline - Durkheim et Lévy-Bruhl - étaient, comme vous, juifs...

CLS : Vous pourriez ajouter Boas et Mauss, mais aussi un nombre beaucoup plus élevé d'ethnologues non juifs, Morgan, Tylor et, plus près de nous, Rivet, Leenhardt, Rivers, Malinowski, Kroeber, Radcliffe-Brown, Evans-Pritchard, Linton, Murdock, Mead... J'admets qu'en sociologie et en ethnologie on compte une proportion notable de juifs. Peut-être ne faut-il pas y attacher plus d'importance qu'à la proportion notable de noms doubles parmi les ethnologues. Il me semble, toutefois, qu'on peut proposer deux explications. En premier lieu, la promotion sociale des juifs, au XIX ${ }^{\mathrm{e}}$ siècle, a coïncidé avec la constitution des sciences sociales comme 
disciplines de plein droit. Il y avait donc là une «niche »-au sens écologique du terme - en partie vacante et où de nouveaux venus pouvaient s'établir sans se heurter à une trop rude compétition. En second lieu, il faut considérer les effets psychologiques et moraux de l'antisémitisme dont, comme tant d'autres, j'ai fait, dès l'enfance, l'expérience intermittente à l'école primaire et au lycée. Se découvrir subitement contesté par une communauté dont on croyait être partie intégrante peut conduire un jeune esprit à prendre quelque distance à l'égard de la réalité sociale, contraint qu'il est de la considérer simultanément du dedans où il la sent et du dehors où on le met.

Voir aussi Tobie Nathan, "Quelques grandes voix juives en matière d'anthropologie et d'ethnopsychiatrie », conférence lors de la journée de la francophonie «Les grandes voix juives francophones ", 19 mars 2009, Collège académique de Netanya, http:// www.ethnopsychiatrie.net/Gdesvoixjves.htm.

2. Cette question perdure encore aujourd'hui sous la forme de la "spécificité de l'État d'Israël comme objet d'étude». Voir par exemple les débats et les conférences organisés à l'Université de Brandeis.

3. Récemment encore, Leon Wieseltier ne reconnaît pas la légitimité de l'étude du corps dans le judaïsme : «La vie du juif se concevait comme une grande saga de l'engagement entre forme et matière, entre corps et âme. [...] Le Juif était une unité psychosomatique », Jewish Quarterly Review, 95/5, 2005, p. 437.

4. «Autour de la Pensée sauvage. Réponses à quelques questions, Entretien du "groupe philosophique" Esprit avec Claude Lévi-Strauss (novembre 1963)», Esprit 322, 1963, p. 628-653, http://www.fondsricoeur.fr/photo/pensee\%20sauvage(1).pdf. Voir aussi le dossier "Claude Lévi-Strauss, une anthropologie "bonne à penser" ", Esprit, $\mathrm{n}^{\circ}$ 1, 2004.

5. Claude Lévi-Strauss, «De la fidélité au texte», L'Homme, vol. 27, nº 101, 1987, p.117-140. (http://www.persee.fr/web/revues/home/prescript/article/

hom_0439-4216_1987_num_27_101_368769)

6. Claude Lévi-Strauss, «Exode sur Exode », L'Homme, vol. 28, nº 106-107, 1988, p. 13-23. (http:// www.persee.fr/web/revues/home/prescript/article/hom_0439-4216_1988_num106_368967)

7. Claude Lévi-Strauss, Mythologiques 1 : Le Cru et le cuit, Paris, Plon, 1964, réimpression 2001.

8. Entretien Esprit p. 7.

9. Ibid. p. 3.

10.

Des mythes "historisés", nous en connaissons beaucoup par le monde ; il est tout à fait frappant, par exemple, que la mythologie des Indiens Zuni du sud-ouest des États-

Unis ait été 'histor théologiens mythes des a votre étude, ne ti mythes austra Ibid.p. 6.

11. Mais voir par exemple une opinion très proche chez S. R. Driver, The Book of Genesis, Londres, Methuen, 1926, p. 83: "As a rule, the Hebrew narrators stripped off the mythological colouring of the pieces of the folklore which they record ; but in the presence instance it is still discernible."

12. Ibid. p. 3.

13. Pierre Clastres, La Société contre l'État, Paris, Minuit, 1974.

14. Entretien Esprit, p. 8.

15. Voir par exemple David Jobling, The Sense of Biblical Narrative: Structural Analysis in the Hebrew Bible, Sheffield, 1986 ; David M. Gunn, The Fate of King Saul. An Interpretation of a Biblical Story, Sheffield, 1980 ; Aharon H. Fish, "Eldad et Medad prophétisent dans le camp. Analyse structuraliste de Nombres 11 », in Uriel Simon (éd.), Studies in Bible Exegesis Presented to Yehuda Elitzur, Ramat Gan, 1986, pp. 45-55 (en hébreu). 
16. Entretien Esprit, p. 4.

17. Michael P. Carroll, "A Structuralist Exercise: the Problem of Moses' Name", American Ethnologist 12 (4), pp. 775-778.

18. «De la fidélité au texte », p. 132.

19. Marcel Détienne, L'invention de la mythologie, Paris, Gallimard, 1981.

20. Pierre Vidal-Naquet, Le chasseur noir. Formes de pensée et formes de société dans le monde grec, $1^{\mathrm{re}}$ éd. Paris, François Maspero, 1981 ; éd. utilisée Paris, La Découverte, 2005, « Avant-propos », p. 14.

21. Mary Douglas, Purity and Danger: An Analysis of the Concepts of Pollution and Taboo, Londres, 1966

(trad. fr. De la souillure. Essai sur les notions de pollution et de tabou , Paris, Maspero, 1971) ; "The Forbidden Animals in Leviticus",

Journal for the Study of the Old Testament 59, 1993, pp. 3-23; In the Wilderness.

The Doctrine of Defilement in the Book of Numbers. JSOT Sup.Ser.158, 1993, Sheffield, JSOT Press.

22. Le Cru et le cuit, p. 338.

23. Ibid.p. 61.

24. Ibid.p. 287.

25. Ibid.p. 338.

26. « Exode surExode », p. 19-20.

27. C'est en ces termes que Lévi-Strauss décrit la situation, « Exode surExode », p. 14 : « $[\ldots]$ "scène mystérieuse"

on d'une tradition archaïque, aurait

(Dhorme 1956, 186) ; "le plus énigmatique de tous nos documents [sur l'initiation]. Il apparaît comme un 'bloc erratique' mal rattaché au contexte" (Caquot 1965, 130). L'épisode, vestige croitLa Bible. L'Ancien Testament

, Tome I. Introduction, traduction et notes par Édouard Dhorme, Paris, Gallimard, « Bibliothèque de la Pléiade », 1956 et

André Caquot, « Pour une étude de l'initiation dans l'ancien Israël »,

Studies in the History of Religions

(Supplements to Numen X: Initiation), Leiden, E. J. Brill, 1965, pp. 119-133.

28. Ibid. p. 20.

29. Nous insérons ici une longue citation de Lévi-Strauss, " Exode sur Exode », p. 15, qui éclaire ce point. L'auteur renvoie entre parenthèses, à de nombreuses références bibliographiques que nous précisons par la suite.

"Les hébraïsants nous enseignent que du verbehâtan"circoncire" dérivent les motshôtên

"beau-père",hâtân

“fiancé, gendre, époux" (Dhorme 1956, 186 ; Caquot 1965, 131. Cf. Hastings 1928, III : 66Ib, 664a ;

Encyclopédie de l'Islam, 1986, art.khitâri

). Des exégètes en concluent qu'à l'origine, il incombait au père et au frère aîné d'une femme de ci : le rite établirait la relation de pa circoncire le fils de celle-

i-orubadari

, 'ami rituel' ou parrain du novice, qui lui impose l'étui pénien, est toujours membre du clan du père (EB, I : 639 ; Crocker 1979 : 285). En raison de l'organisation dualiste, ce clan relève de la moitié où le jeune homme prendra obligatoirement femme : "C'est le

i-orubadari

qui impose au novice l'étui pénien, objet associé à des droits catégoriques sur la sexualité des femmes de l'autre moitié [...]. Le lien du parrain avec le novice ne procède pas de quelque communauté de substance, mais d'une relation générale, catégorique, de complémentarité logique telle qu'on la trouve dans la parenté par alliance." (Crocker, ibid. : 286). L'ethnologue se rencontre donc avec l'hébraïsant qui, lui aussi, met en rapport direct circoncision et mariage : "II reste à préciser ce qu'est un 'mari de sang'. L'interprétation la plus simple consiste à y voir un époux ou un fiancé capable de verser le sang, c'est-à-dire de déflorer une vierge" (Caqu 
La Bible. L'Ancien Testament

, Tome I. Introduction, traduction et notes par Édouard Dhorme, Paris, Gallimard, « Bibliothèque de la Pléiade », 1956.

André Caquot, « Pour une étude de l'initiation dans l'ancien Israël »,

Studies in the History of Religions

133.

(Supplements to Numen X: Initiation), Leiden, E. J. Brill, 1965, pp. 119-

Jon Christopher Crocker, “Selves and Alters among the Eastern Bororo”, in David Maybury-Lewis (éd.), Dialectical Societies: The Gê and Bororo of Central Brazil

300.

, Cambridge, Harvard University Press, 1979, pp. 249-

César Albisetti \& Angelo Jayme Venturelli,Enciclopedia Bororo

, Campo Grande, Mato Grosso, Brasil (« Publicaçoes 1, 2, 5 do Museu Regional Dom Bosco »), 3 vol.,

1962-1976.

James Hastings (éd.),Encyclopaedia of Religion and Ethics

, III, New York, Charles Scribner's Sons, 1928.

30. Ibid. p. 19.

31. Ibid. p. 17.

32. Ibid. p. 20.

33. Ibid. p. 15 et 20 .

34. Voir l'article de Daniel Boyarin.

35. « Exode sur Exode », p. 21.

36. Ibid.p. 15.

37. Ibid. p. 21.

38. Ibid. p. 20.

39. Ibid. p. 20.

40. Ibid. p. 21.

41. Fernando Giobellina Brumana, Elda Evangelina González, « Mito: Rito : Lévi-Strauss: Mary Douglas », Revista española de antropología americana $\mathrm{n}^{\circ} 11,1981$, p. 245-258.

42. Voir par exemple le numéro spécial du Journal of Hebrew Scriptures, vol. 8, 2008, http:// www.jhsonline.org; les commentaires de Jacob Milgrom sur le Lévitique: Leviticus 1-16, New York, Anchor Bible, 1998 ; Leviticus 17-22, New York, Anchor Bible, 2000 ; Leviticus 23-27, New York, Anchor Bible, 2000 ; Leviticus: A Book of Ritual and Ethics, Minneapolis, Fortress Press, 2004 ; John F. A. Sawyer, Reading Leviticus: Responses to Mary Douglas, T\& T Clark, 1996 ; Jean Duhaime, «Lois alimentaires et pureté corporelle dans le Lévitique. L'approche de Mary Douglas et sa réception par Jacob Milgrom », Religiologiques 17, printemps 1998, dossier « Nourriture et sacré », p. 19-35 et de nombreux autres travaux.

\section{RÉSUMÉS}

Comment expliquer l'exclusion du judaïsme antique du domaine de l'anthropologie historique? Parmi ces réponses, une retient en particulier notre attention, à savoir celle qui évoque la spécificité du judaïsme comme objet d'étude. C'est dans cette lignée que s'inscrit la position de Claude Lévi-Strauss. Paradoxalement, il justifie théoriquement l'écart des sources hébraïques 
anciennes de son analyse structuraliste et en même temps, inspire un certain nombre de travaux qui mettent à profit la méthode structuraliste dans l'étude des textes du judaïsme ancien. Le présent article se propose de présenter ce dossier, celui du rapport de Lévi-Strauss aux sources juives/hébraïques anciennes.

How is to explain the fact that the field of historical anthropology did not include ancient Judaism among its' topics of interest. The answer retaining here our attention emphasizes the specificity of Judaism and Claude Lévi-Strauss belongs to this school of thought. Paradoxically, the father of cultural anthropology argues against the application of the structural method to the ancient Hebrew sources and in the same time inspired some of the major anthropological approaches to ancient Judaism, such as Mary Douglas' for example. The present paper aims to analyze the relationship of Lévi-Strauss to ancient Hebrew/Jewish sources as revealed in four of his writings.

INDEX

\section{מילות מפתח}

קלוד לוי שטראוס, הרמנויטיקה, סטרוקטוראליזם, יהדות, מילה, השוואתיות, המזרח:

התיכון, הים התיכון

Keywords : Lévi-Strauss Claude (1908-2009), structuralism, hermeneutics, Judaism, circumcision, comparatism, Mediterranean, Middle East

Index géographique : Méditerranée, Proche-Orient

Mots-clés : Lévi-Strauss Claude (1908-2009), herméneutique, structuralisme, judaïsme, circoncision, comparatisme 